High Energy Phenomena in Relativistic Outflows (HEPRO IV)

International Journal of Modern Physics: Conference Series

Vol. 28 (2014) 1460170 (6 pages)

(C) The Author

DOI: 10.1142/S2010194514601707

\title{
EVOLUTION OF PLASMOID-CHAIN IN POYNTING-DOMINATED PLASMA*
}

\author{
MAKOTO TAKAMOTO \\ Max-Planck-Institut für Kernphysik, Heidelberg, Germany \\ makoto.takamoto@mpi-hd.mpg.de
}

Received 30 October 2013

Revised 3 December 2013

Published 21 March 2014

\begin{abstract}
We present our recent results of the evolution of the plasmoid-chain in a Poynting dominated plasma. We model the relativistic current sheet with cold background plasma using the relativistic resistive magnetohydrodynamic approximation, and solve its temporal evolution numerically. Numerical results show that the initially induced plasmoid triggers a secondary tearing instability. We find the plasmoid-chain greatly enhances the reconnection rate, which becomes independent of the Lundquist number, when this exceeds a critical value. Since magnetic reconnection is expected to play an important role in various high energy astrophysical phenomena, our results can be used for explaining the physical mechanism of them.
\end{abstract}

Keywords: Magnetic fields; magnetohydrodynamics (MHD); relativistic processes; plasmas.

PACS Numbers: 52.35.-g, 95.30.Qd, 52.30.-q, 52.27.Ny, 52.35.Vd

\section{Introduction}

Magnetic reconnection is a process that converts magnetic field energy into thermal and kinetic energy very efficiently. Because of this, it is believed that magnetic reconnection plays an important role in various phenomena from the laboratory plasma to the astrophysical plasma. Recently, interest in the properties of relativistic magnetic reconnection has been growing, especially in Poynting-dominated plasmas, which are believed to be present in various high energy astrophysical phenomena. In those models, the Poynting energy of the plasma is assumed to be dissipated into thermal and kinetic energy almost completely at some distance from the central object. However, such an efficient dissipation process is still unknown. In the

* This is an Open Access article published by World Scientific Publishing Company. It is distributed under the terms of the Creative Commons Attribution 3.0 (CC-BY) License. Further distribution of this work is permitted, provided the original work is properly cited. 
last decade, several studies have been performed with the goal of finding efficient dissipation processes. ${ }^{1-4}$ Magnetic reconnection is one of the most promising candidates among them, and has been studied actively from analytical and numerical points of view. Although the typical timescale of this process is much shorter than the resistive time scale, it is still too slow to explain observed timescale of many astrophysical phenomana. Recently, it was found that spontaneous current sheet fragmentation in a non-relativistic plasma occurs via secondary tearing instabilities when the Lundquist number exceeds a critical value, leading to the so-called plasmoid-chain. The critical value is thought to be about $10^{4}$ in the non-relativistic plasma, ${ }^{5-7}$ and it also results in fast reconnection rate.

In this paper, we investigate the evolution of the plasmoid-chain in a cold Poynting-dominated background plasma with large Lundquist number: $S_{L} \sim 10^{3}-$ $10^{5}$. We also investigate statistical properties of the plasmoid-chain, such as the distribution function of the plasmoid width. To study the evolution of the secondary tearing instability, we use a uniform, constant resistivity, and initialize the magnetic field with a perturbations localized at the origin. This enables us to understand the evolution of current sheets in which a tearing instability is triggered at a point.

\section{Evolution of Plasmoid-Chain}

Current sheets are unstable to the tearing instability whose typical growing timescale can be written as:

$$
\tau_{\max }=\sqrt{\tau_{R} \tau_{A}}=\left(\frac{\delta}{c_{A}} \frac{\delta^{2}}{\eta}\right)^{1 / 2},
$$

where $\tau_{R} \equiv \delta^{2} / \eta$ is the resistive diffusion timescale and $\tau_{A} \equiv \delta / c_{A}$ is the Alfvén crossing time across a current sheet, $\delta$ is the sheet thickness, $\eta$ is the resistivity, and $c_{A}$ is the Alfvén velocity in the background plasma. ${ }^{8,9}$ This shows that the tearing instability grows faster as the sheet width $\delta$ becomes small. When a plasmoid grows along a current sheet, the current sheet behind plasmoids shrinks and this triggers the growth of other small plasmoids. If we approximate a sheet thickness behind plasmoids using the Sweet-Parker sheet, $\delta \sim L / \sqrt{S_{L}}$ where $L$ is the sheet length and $S_{L} \equiv L c_{A} / \eta$, Eq. (1) can be rewritten as follows.

$$
\tau_{\max } \sim \frac{\tau_{A, L}}{S_{L}^{1 / 4}},
$$

where $\tau_{A, L}=L / c_{A}$ is the Alfvén crossing time along the sheet. This indicates that the growth of the tearing instability becomes very fast when $S_{L}$ becomes larger than $10^{4}$, which can be considered as the critical Lundquist number of the plasmoid-chain. $S_{L}$ depends on the current sheet length $L$ and this means current sheets will be filled with a plasmoid-chain when the sheet length is sufficiently long.

A more complete derivation is presented in Refs. 7, 10, 11. 


\section{Numerical Setup}

We model a current sheet using the relativistic resistive magnetohydrodynamic (RRMHD) approximation. We solve it using a RRMHD numerical scheme developed by Ref. 12 which preserves the divergence free constraint on the magnetic field using the constrained transport algorithm. ${ }^{13}$ We calculate the RRMHD equations in a conservative fashion, and used the relativistic ideal equation of state.

We prepare a square domain which is very long along the current sheet. and divide it into homogeneous numerical meshes with size $\Delta=5 \delta / 128 \sim 0.04 \delta$. For the initial condition, we consider the static relativistic Harris current sheet: ${ }^{14}$

$$
\begin{aligned}
B_{z}(x) & =B_{0} \tanh (x / \delta), \\
p(x) & =p_{\text {in }}+p_{s} / \cosh ^{2}(x / \delta), \\
\rho(x) & =\rho_{\text {in }}+\rho_{s} / \cosh ^{2}(x / \delta),
\end{aligned}
$$

where $p, \rho$ are the gas pressure and the rest mass density, and other variables are set to 0 except for a small perturbation of the magnetic field described later. For the upstream region of the current sheet, we consider a cold plasma $p_{s}=0.1 \rho_{s}$; for the inside of the sheet, we consider a relativistically hot plasma $\rho_{s}=p_{s}$ where $p_{s}=B_{0}^{2} / 8 \pi$. Note that the temperature of the sheet decreases with decreasing magnetic field strength. In this calculation, we use a constant resistivity. To trigger the initial tearing instability at the origin $(x, z)=(0,0)$, we add the following small perturbation to the magnetic field:

$$
\delta A_{y}=-0.03 B_{0} \delta \exp \left[-\left(x^{2}+z^{2}\right) / 4 \delta^{2}\right] .
$$

To model magnetic reconnection in high energy astrophysical phenomena, we consider magnetically dominated plasma with magnetization parameter $\sigma_{i n}=14$.

\section{Results}

Figure 1 shows a snapshot of a pressure profile at the time when the largest plasmoid to result from the initial perturbation reaches the edge of numerical domain. Since plasmoids move at approximately the Alfvén velocity of the upstream flow unless the plasmoid inertia is comparable to the magnetic field energy, the escape time is of the order of $t_{A}$. We find that many plasmoids evolve along the current sheet.

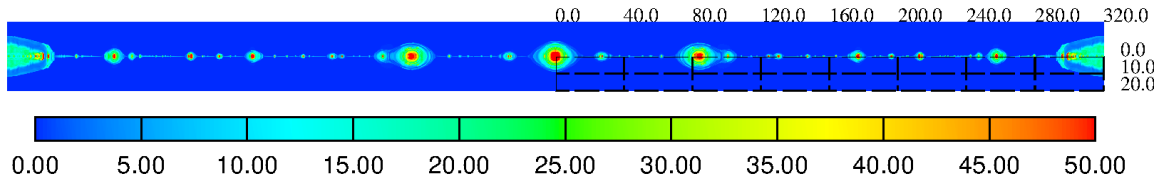

Fig. 1. Snapshots of the pressure profile just before the largest plasmoid run away from the numerical domain. 

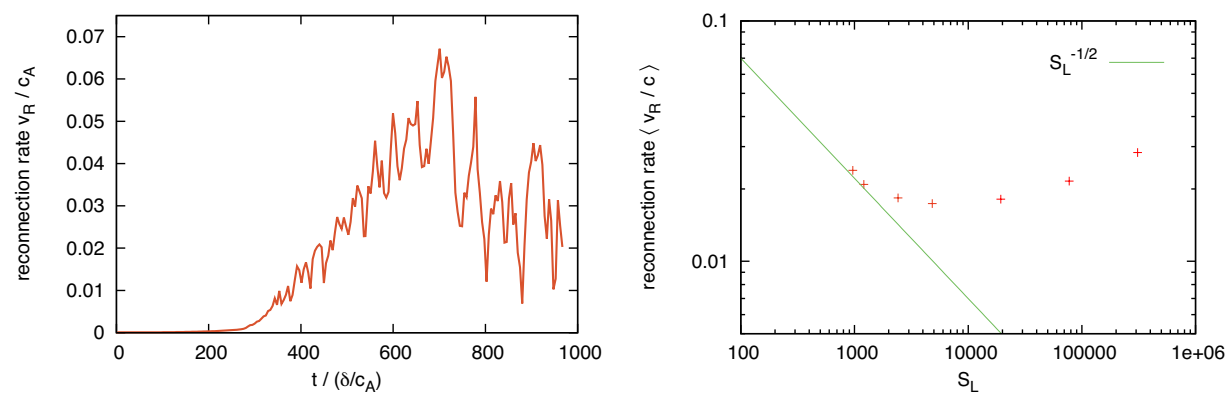

Fig. 2. Left: The temporal evolution of the reconnection rate. Right:The plot of the time averaged reconnection rate $\left\langle v_{R} / c_{A}\right\rangle$ over the statistical equilibrium region with respect to the Lundquist number $S_{L}$.

As we mentioned in the previous section, the evolution of a plasmoid induces a thinner current sheet behind it, leading to a secondary tearing instability and the generation of a the plasmoids-chain.

Left panel of Fig. 2 shows the time evolution of the reconnection rate in units of the Alfvén crossing time, $L_{z} / c_{A} \equiv t_{A}$. The reconnection rate is defined as:

$$
v_{R} / c_{A} \equiv-\frac{c}{B_{0} c_{A} L_{z}} \int_{0}^{L_{z}} d z E_{y}(x=0, z) .
$$

The reconnection rate grows until the largest plasmoid, which is initially triggered at the origin, escapes from the numerical domain, at which point the reconnection rate has increased up to $\sim 0.05 c_{A}$. After this, the plasmoid-chain reaches a statistical steady state and the averaged reconnection rate is about $0.03 c_{A}$, which is approximately twice that of the relativistic tearing instability without a plasmoidchain. ${ }^{15}$

Right panel of Fig. 2 is the time-averaged reconnection rate $\left\langle v_{R} / c_{A}\right\rangle$ as a function of the Lundquist number $S_{L}$. We calculate the time average of the reconnection rate curves over the plateau region where the plasmoid-chain reaches a statistical equilibrium state. As in the non-relativistic case, we find that the reconnection rate becomes independent of the Lundquist number when it is larger than a critical value $S_{c}$. For small Lundquist numbers, we find the Sweet-Parker sheet dependence $S_{L}^{-1 / 2}$ of the reconnection rate predicted by Refs. 16 and 17 . In our calculations, the critical value is $S_{c} \sim 2-3 \times 10^{3}$, which is a little less than that of the weak magnetic field case. As pointed out by Ref. 7 , the reconnection rate of the plasmoid-chain can be written as, $v_{R} / c_{A} \sim 1 / \sqrt{S_{c}}$, using the relation of the Sweet-Parker sheet. If we use the above critical values, $S_{c}=3 \times 10^{3}$, in the strongly magnetized case, the reconnection rate is $\sim 0.02 c_{A}$, which agrees with the values indicated in the top panel of Fig. 2.

In Fig. 3, we plot the time-averaged distribution functions after the initially triggered plasmoids escaped. In the small plasmoid region, the distribution function has an index of -2 as predicted by previous works for the non-relativistic case. ${ }^{7}$ In 


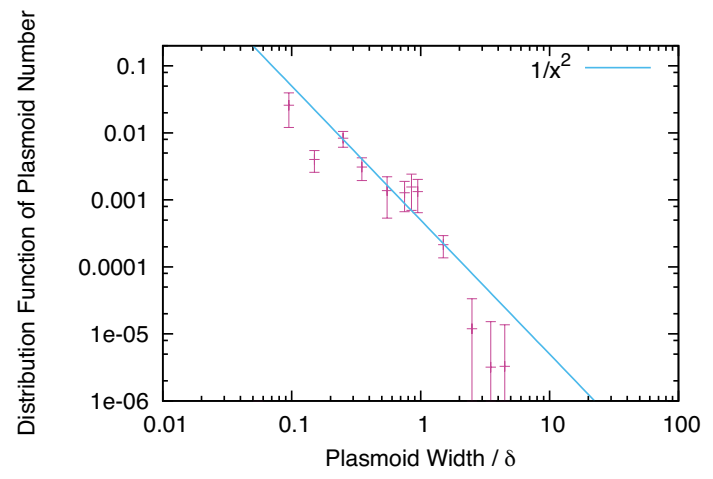

Fig. 3. The time-averaged distribution of plasmoid size perpendicular to the current sheet. The distribution functions are averaged over after $t=2.2 t_{A}$.

the larger plasmoid region, $w>\delta$, the distribution drops rapidly. We consider this is due to the effect of the plasmoid loss by advection. Since the initially triggered plasmoid already escaped from the simulation domain in this case, the plasmoids can freely escape from the domain and this results in the exponential decay of the distribution function.

\section{Summary}

In this paper, we investigated the evolution of the plasmoid-chain in a high- $\sigma$ plasma. We modeled the relativistic current sheet with cold background plasma using the relativistic resistive magnetohydrodynamic approximation, and solved its temporal evolution numerically. We performed various calculations using large magnetization parameter and different Lundquist numbers with respect to the sheet length from $S_{L} \sim 10^{3}$ to $S_{L} \sim 10^{5}$. The numerical results show that the initially induced plasmoid triggers a secondary tearing instability and the current sheet is gradually filled with many plasmoids, that is, it evolves into a plasmoid-chain, as predicted by nonrelativistic work. As expected, this plasmoid instability enhances the reconnection rate, which grows until the initially triggered plasmoid escapes from the simulation domain, reaching up to $\sim 0.05 c_{A}$. Subsequently, the plasmoid-chain reaches a statistically equilibrium state, and the temporally averaged reconnection rate in a steady state becomes $\sim 0.03 c_{A}$. Since the maximum value of the Alfvén velocity is the light velocity $c$, our numerical calculation indicates the maximum reconnection rate of the plasmoid-chain is $0.03 c$.

We also investigated the plasmoid size distribution. Our numerical results show that in strongly magnetized cases the distribution becomes power law with index -2 in the small plasmoid region. This indicates that the plasmoid loss is mainly due to mergers. In large plasmoid region, the distribution function shows exponential decay because of the free advective escape of plasmoids from the domain. 


\section{Acknowledgments}

Numerical computations were carried out on SR16000 at YITP in Kyoto University. Calculations were also carried out on the Cray XT4 at Center for Computational Astrophysics, CfCA, of National Astronomical Observatory of Japan. This work is supported by Max-Planck-Institut für Kernphysik and the Postdoctoral Fellowships for Research Abroad program by the Japan Society for the Promotion of Science No. 20130253 (M. T.).

\section{References}

1. A. Lazarian and E. T. Vishniac, Astrophysical Journal 517, 700 (June 1999).

2. M. Takamoto, T. Inoue and S. Inutsuka, Astrophysical Journal 755, p. 76 (August 2012).

3. T. Amano and J. G. Kirk, Astrophysical Journal 770, p. 18 (June 2013).

4. I. Mochol and J. G. Kirk, Astrophysical Journal 771, p. 53 (July 2013).

5. K. Shibata and S. Tanuma, Earth, Planets, and Space 53, 473 (June 2001).

6. N. F. Loureiro, A. A. Schekochihin and S. C. Cowley, Physics of Plasmas 14, p. 100703 (October 2007).

7. D. A. Uzdensky, N. F. Loureiro and A. A. Schekochihin, Physical Review Letters 105, p. 235002 (December 2010).

8. H. P. Furth, J. Killeen and M. N. Rosenbluth, Physics of Fluids 6, 459 (1963).

9. B. C. Low, Astrophysical Journal 181, 209 (April 1973).

10. A. Bhattacharjee, Y.-M. Huang, H. Yang and B. Rogers, Physics of Plasmas 16, p. 112102 (November 2009).

11. N. F. Loureiro, A. A. Schekochihin and D. A. Uzdensky, Phys. Rev. E 87, p. 013102 (January 2013).

12. M. Takamoto and T. Inoue, Astrophysical Journal 735, p. 113 (July 2011).

13. C. R. Evans and J. F. Hawley, Astrophysical Journal 332, 659 (September 1988).

14. F. C. Hoh, Physics of Fluids 9, 277 (February 1966).

15. H. R. Takahashi, T. Kudoh, Y. Masada and J. Matsumoto, Astrophysical Journal, Letters 739, p. L53 (October 2011).

16. M. Lyutikov and D. Uzdensky, Astrophysical Journal 589, 893 (June 2003).

17. Y. E. Lyubarsky, MNRAS 358, 113 (March 2005). 